\title{
Morphological Analysis of Bone Deformities of the Distal Femur in Toy Poodles with Medial Patellar Luxation
}

\author{
Shinji Yasukawa ${ }^{1,2,3}$ Kazuya Edamura $^{1}$ Koji Tanegashima ${ }^{1}$ Mamiko Seki ${ }^{1}$ Kazushi Asano $^{1}$ \\ Tomohiro Nakayama ${ }^{4}$ Kei Hayashi ${ }^{5}$
}

${ }^{1}$ Laboratory of Veterinary Surgery, Department of Veterinary Medicine, College of Bioresource and Sciences, Nihon University, Fujisawa, Kanagawa, Japan

2 ONE for Animals, Yokohama, Kanagawa, Japan

${ }^{3}$ Department of Orthopedic Surgery, DVMs Animal Medical Center Yokohama, Yokohama, Kanagawa, Japan

${ }^{4}$ Laboratory of Veterinary Radiology, Department of Veterinary Medicine, College of Bioresource and Sciences, Nihon University, Fujisawa, Kanagawa, Japan

${ }^{5}$ Department of Clinical Sciences, College of Veterinary Medicine, Cornell University, Ithaca, New York, United States

\author{
Address for correspondence Kazuya Edamura, PhD, DVM, Diplomate \\ JCVS, Laboratory of Veterinary Surgery, Department of Veterinary \\ Medicine, College of Bioresource Sciences, Nihon University, \\ 1866 Kameino, Fujisawa, Kanagawa 252-0880, Japan \\ (e-mail: edamura.kazuya@nihon-u.ac.jp).
}

Vet Comp Orthop Traumatol 2021;34:303-311.

\begin{abstract}
Keywords

- computed tomography

- dogs

- hypoplasia

- patellar luxation

- trochlear groove

Objective The aim of this study was to evaluate the bone deformities of the distal femur of Toy Poodles with medial patellar luxation (MPL) using computed tomography. Methods Computed tomography data from 37 stifles of Toy Poodles (16 normal, 10 grade $2 \mathrm{MPL}$ and 11 grade $4 \mathrm{MPL}$ ) were analysed. For trochlear morphology, sulcus angle, medial or lateral trochlear inclination and trochlear angle were compared. For condylar morphology, distance between the apex of the medial or lateral trochlear ridge and the most caudal point of the medial or lateral femoral condyle (MC or LC), the length of the cranial/middle (anterior) part (MC(a) or $\mathrm{LC}\left(\mathrm{a}^{\prime}\right)$ ) and caudal (posterior) part (MC(p) or LC(p')) of distal femur and medial or lateral condyle width (MCW or LCW) were measured, and the ratios of medial-to-lateral condyles and anterior-posterior parts were compared.

Results Sulcus angle was significantly higher, and medial trochlear inclination was significantly lower in grade 4 MPL group than other groups. Lateral trochlear inclination was not significantly different among 3 groups. Trochlear angles at proximal trochlear groove were significantly lower in grade $4 \mathrm{MPL}$ group than other groups. MC/LC, MC(a)/ $\mathrm{LC}\left(\mathrm{a}^{\prime}\right), \mathrm{MC}(\mathrm{a}) / \mathrm{MC}(\mathrm{p})$, and $\mathrm{LC}\left(\mathrm{a}^{\prime}\right) / \mathrm{LC}\left(\mathrm{p}^{\prime}\right)$ were significantly lower in grade $4 \mathrm{MPL}$ group than other groups; however, there were no significant differences in $\mathrm{MC}(\mathrm{p}) / \mathrm{LC}\left(\mathrm{p}^{\prime}\right)$ and MCW/LCW among three groups.

Conclusion Hypoplasia of the cranial and middle rather than caudal compartment of the distal femur was the primary morphological abnormality in Toy Poodles with grade $4 \mathrm{MPL}$.
\end{abstract}

received

June 27, 2020

accepted after revision

January 26, 2021

published online

May 12, 2021 (c) 2021. Thieme. All rights reserved. Georg Thieme Verlag KG,

Rüdigerstraße 14,

70469 Stuttgart, Germany
DOI https://doi.org/ 10.1055/s-0041-1726084. ISSN 0932-0814. 


\section{Introduction}

Medial patellar luxation (MPL) is one of the most common orthopaedic disorders in dogs. ${ }^{1-4}$ It has been reported that severe MPL is associated with numerous bone deformities including varus deformity of the distal one-third of the femur, lateral torsion of the distal femur, shallow trochlear groove with poorly developed or absent medial trochlear ridge and hypoplasia of the medial condyle. ${ }^{1,3,5}$ Traditionally, these bone deformities have been evaluated using plain radiographs. ${ }^{6}$ For example, lateral and skyline views have been used to evaluate the depth of the trochlear groove and femoropatellar congruence. ${ }^{7-10}$ However, evaluation of the trochlear groove by these views can be challenging because of the difficulty in obtaining a proper radiographic position, ${ }^{11}$ and no adequate anatomical landmark has yet been established. Recently, computed tomography (CT) has been increasingly used to evaluate bone abnormalities in dogs. ${ }^{11-13}$ In 2016, Yasukawa and colleagues reported that Toy Poodles with grade 4 MPL had significant femoral varus deformity, medial displacement of the tibial tuberosity, internal rotation of the proximal tibia and hypoplasia of the patella as observed using three-dimensional (3D) CT. ${ }^{14}$ However, the depth of the trochlear groove and the morphology of the femoral condyles were not evaluated objectively due to lack of appropriate landmarks.

The current study aimed to establish objective methods to evaluate the morphology of the trochlear groove and femoral condyles in dogs with MPL. To the best of our knowledge, only one previous study has evaluated the depth of the femoral trochlear groove in dogs with MPL using CT. ${ }^{11}$ Morphological evaluation of the trochlear groove and femoral condyle may contribute to greater understanding of the pathogenesis of canine MPL. Furthermore, objective data may help to establish the criteria for indication of trochleoplasty, which is often determined by subjective visual inspection following arthrotomy and the surgeon's experience. We hypothesized that quantitative morphological abnormalities of the distal femur would be more severe in stifles with MPL. The objectives of this study were to determine the morphological parameters of the distal femur (trochlear groove and the femoral condyle) using CT and to compare these parameters among stifles without MPL, with grade 2 MPL and with grade $4 \mathrm{MPL}$ to characterize the morphological abnormalities associated with MPL.

\section{Materials and Methods}

\section{Case Selection}

Computed tomography data were obtained from both hindlimbs of Toy Poodles with a clinical diagnosis of unilateral or bilateral MPL (MPL groups). Computed tomography data of the hindlimbs of Toy Poodles with medical conditions (such as humeral fracture, cervical disease, carpal luxation and femoral head necrosis on the contralateral limb) without any stifle pathology were used as the normal group. The dogs were presented to Animal Medical Center, Nihon University (Kanagawa, Japan) between
April 2012 and July 2014, and consent for performing diagnostic CT was obtained from all owners. The use of CT data for this study was approved by the owners and the institutional committee. The hindlimbs were classified as normal or grade 1 to $4 \mathrm{MPL}$ according to the Singleton grading system, ${ }^{15}$ and normal stifles and stifles with grades 2 and 4 MPL were included. Hindlimbs with grade 1 or 3 MPL, lateral patellar luxation, or hip and tarsal joint diseases were excluded.

\section{CT Imaging Protocol}

All CT images were acquired on a 16-line helical scanner (Aquilion LB 16 Slice, Toshiba Medical Systems, Otawara, Japan) and were reconstructed as 3D images using image processing software (AZE VirtualPlace, AZE Co., Ltd, Tokyo, Japan). All investigated dogs were premedicated subcutaneously with atropine sulphate (Atropine sulfate injection, Mitsubishi Tanabe Pharma Corporation, Osaka, Japan) (0.04 $\mathrm{mg} / \mathrm{kg}$ ) and intravenously with midazolam hydrochloride (Dormicum injection, Astellas Pharma Inc., Tokyo, Japan) $(0.2 \mathrm{mg} / \mathrm{kg})$ and butorphanol tartrate (Vetorphale, Meiji Seika Pharma Co., Ltd., Tokyo, Japan) $(0.2 \mathrm{mg} / \mathrm{kg})$. Anaesthesia was induced with an intravenous injection of propofol (Mylan, Mylan Pharma Co., Ltd., Osaka, Japan) (2.0 to 4.0 $\mathrm{mg} / \mathrm{kg}$ ) and maintained with $1.5 \%$ to $2.0 \%$ isoflurane (Isoflu, DS Pharma Animal Health Co., Ltd., Osaka, Japan) at $100 \%$ oxygen given through an endotracheal tube. Dogs were positioned in dorsal recumbency with the hip, stifle and tarsal joints flexed at approximately 90 degrees. Images were obtained with a $0.5-\mathrm{mm}$ slice thickness and $0.3-\mathrm{mm}$ reconstruction intervals. A bone algorithm was used for image analysis and images were viewed with a window width of 1,000 Hounsfield units (HU) and window level $100 \mathrm{HU}$.

In preparation for CT measurement, the femur was first reconstructed into 3D images, and the surface structures of distal femur were analysed by rotating the reconstructed femur around the short or long axes as shown in - Fig. 1. Then, the most proximal edge of the intercondylar fossa (point 'a' in - Fig. 1A) and the most proximal border of the trochlea groove (point ' $b$ ' in - Fig. 1B) were defined. In the present study, the most proximal portion of the concave on cranial aspect of the distal femur was used as the most proximal border of the trochlea groove (-Fig. 1B). Second, a representative lateral view of the distal femur was obtained. In the present study, the lateral view of the distal femur was defined as an image in which the central one-third of the caudal margins of the medial and lateral condyles were overlapped (red double-headed arrow in - Fig. 1C). Thus, the general lateral view obtained by rotating the frontal view of the femur by 90 degrees was not used in this study to exclude the effects on the varus deformity or lateral torsion of the distal femur. Third, the transverse planes of the distal femur were obtained by slicing the distal femur perpendicular to the line connecting the two points on lateral view (white arrow in -Fig. 1D). Subsequently, 20 transverse planes that sliced with equal widths were generated and numbered from distal to proximal as No.1 to 20 (-Fig. 1E). 


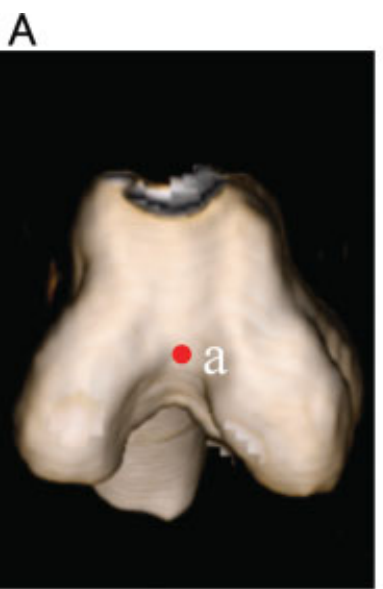

D

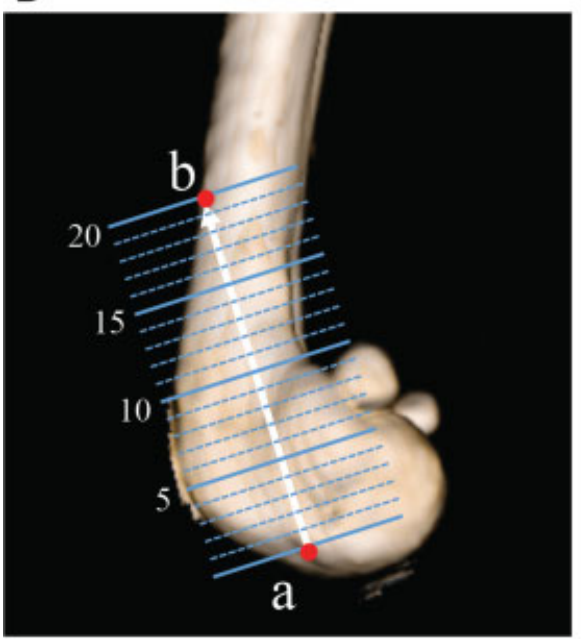

B

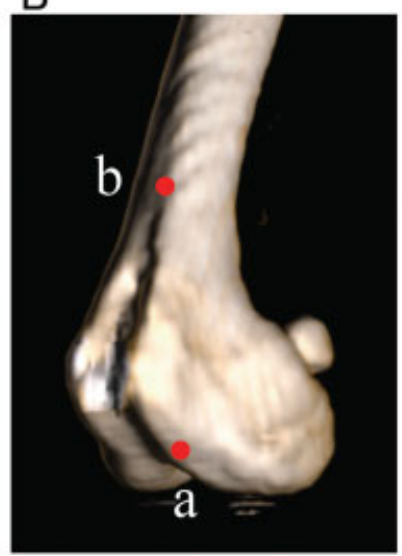

E

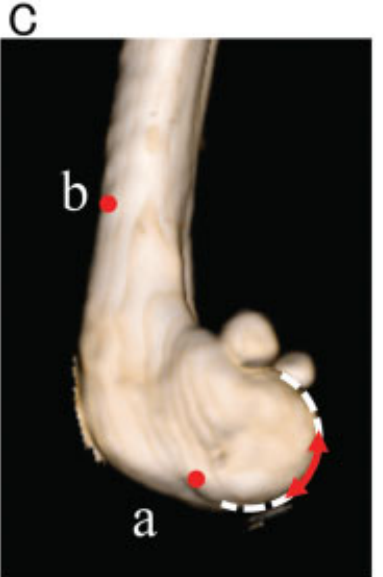

a

Fig. 1 Evaluation method of the morphological findings of the distal femur using three-dimensional computed tomography. (A) Point 'a'. The most proximal edge of the intercondylar fossa was defined as point 'a'. (B) Point ' $b$ '. The most proximal border of the trochlea groove was defined as point ' $b$ '. In the present study, the most proximal portion of the concave on cranial aspect of the distal femur was used as the most proximal border of the trochlea groove. This figure also shows point 'a'. (C) Lateral view of the distal femur. The lateral view of the distal femur was defined as an image in which the central one-third of the caudal margins of the medial and lateral condyles were overlapped (red double-headed arrow). (D) Transverse planes of the distal femur were obtained by slicing the distal femur perpendicular to the line connecting the two points (a and $b$ ) on lateral view. Twenty transverse planes that sliced with equal widths were generated. (E) Transverse planes of the distal femur. These planes were numbered from distal to proximal as No.1 to 20 .

\section{CT Measurements: Trochlear Groove Morphology}

Groove morphology was quantified based on previous human studies. ${ }^{16-18}$ On each transverse plane, the caudal condylar line was drawn at a tangent to the most caudal surfaces of the medial and lateral femoral condyles (dotted green line in - Fig. 2A). A line was drawn (solid green line in -Fig. 2A) parallel to the caudal condylar line, passing the bottom of the trochlear groove (red dot in -Fig. 2A). Sulcus angle (SA) was measured as the angle formed by the medial and lateral trochlear facets and the deepest point of the trochlear groove on each transverse plane (-Fig. 2B)..$^{16,17}$ The medial or lateral trochlear inclination (MTI or LTI) was defined as the angle between the line parallel to the caudal condylar line and passing the bottom of the trochlear groove and the line that was drawn along the surface of the medial or lateral trochlear facets, respectively ( - Fig. 2B). ${ }^{16-18}$ In addition, trochlear angle (TA) was defined as the angle formed by the line that was passing along the apexes of the medial and lateral trochlear ridges on each transverse plane and the line that was parallel to the caudal condylar line on the most distal transverse plane (blue line in - Fig. 2C). ${ }^{16,17}$

\section{CT Measurements: Femoral Condylar Morphology}

Each transverse plane of the distal femur was divided into three compartments for visual presentations: (1) The 'cranial compartment', the area cranial to the bottom of the trochlear groove; (2) the 'caudal compartment', the area caudal to the intercondylar fossa; and (3) the 'middle compartment', the area between the cranial and caudal compartments ( - Fig. 3A). Several parameters were measured to characterize the morphological abnormalities of the distal femur associated with MPL. The distance between the apex of the medial or lateral trochlear ridge and the most caudal point of the medial or lateral femoral condyle (MC or LC) was measured to evaluate the size of the distal femur, including each femoral condyle ( - Fig. 3B). Subsequently, the MC and LC were divided into two parts by the line that was drawn parallel to the caudal condylar line and passing through the intercondylar fossa as follows: 


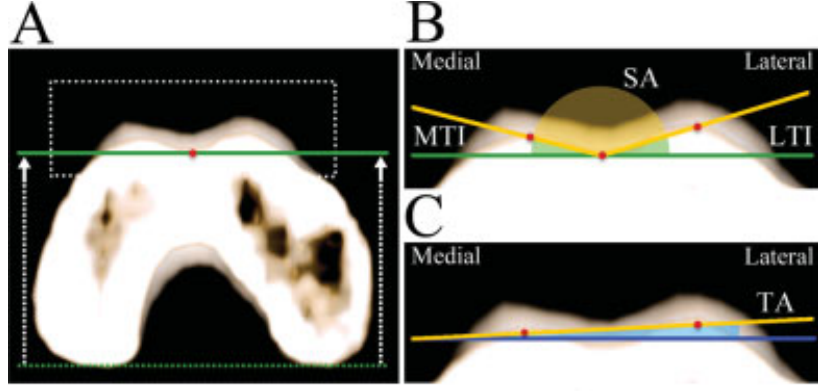

Fig. 2 Measurement values for the cranial compartment of the distal femur. (A) Caudal condylar line: the line drawn at a tangent to the most caudal surfaces of the medial and lateral femoral condyles on each transverse plane (dotted green line). Solid green line was the line that was drawn parallel to the caudal condylar line, passing the bottom of the trochlear groove (red dot). (B) Sulcus angle (SA): the angle formed by the medial and lateral trochlear facets (yellow line) and the deepest point of the trochlear groove. Medial or lateral trochlear inclination (MTI or LTI): the angle between the line parallel to the caudal condylar line and passing the bottom of the trochlear sulcus (solid green line) and the line that drawn along the surface of the medial or lateral trochlear facets (yellow line) respectively. (C) Trochlear angle (TA): the angle formed by the line that was passing along the apexes of the medial and lateral trochlear ridges on each transverse plane (yellow line) and the line that was parallel to the caudal condylar line on the most distal transverse plane (blue line).

The anterior part (cranial comportment and middle compartment; MC (a) or LC (a')) and the posterior part (caudal compartment; MC (p) or LC (p')) ( - Fig. 3C). In this study, 'a' for anterior part (cranial/middle compartments combined) and 'p' for posterior part (caudal compartment) were used to avoid confusion between the various ' $c$ '. The width of the medial or lateral condyle (MCW or LCW) was measured at the level of the line that was drawn parallel to the caudal condylar line and passed through the midpoint of MC (p) or LC (p') ( - Fig. 3D). Then, the ratio of MC/LC, MC (a)/LC (a'), MC (a)/MC (p), LC (a')/LC(p'), MC (p)/LC (p') and MCW/LCW was calculated to evaluate the morphological relationship between the medial and lateral condyles as well as the anterior part (craniomiddle compartments) and posterior part (caudal compartment). All values were obtained using a PACS workstation (Osirix, Osirix Foundation, Lausanne, Switzerland).

\section{Statistical Analysis}

Statistical analyses were performed using a data analysis software package (GraphPad Prism version 6.0 for Macintosh, GraphPad Software Inc., San Diego, California, United States). Normality of the data was tested using the D'Agostino-Pearson omnibus normality test. A one-way analysis of variance was performed to compare the groups. Tukey's multiple comparison was used as the post-hoc test. Values are expressed as mean and standard deviation. Values of $p<0.05$ indicated a significant difference.

\section{Results}

\section{Patients}

Thirty-seven hindlimbs of 27 Toy Poodles were included in this study (16 normal stifles, 10 stifles with grade 2 MPL and
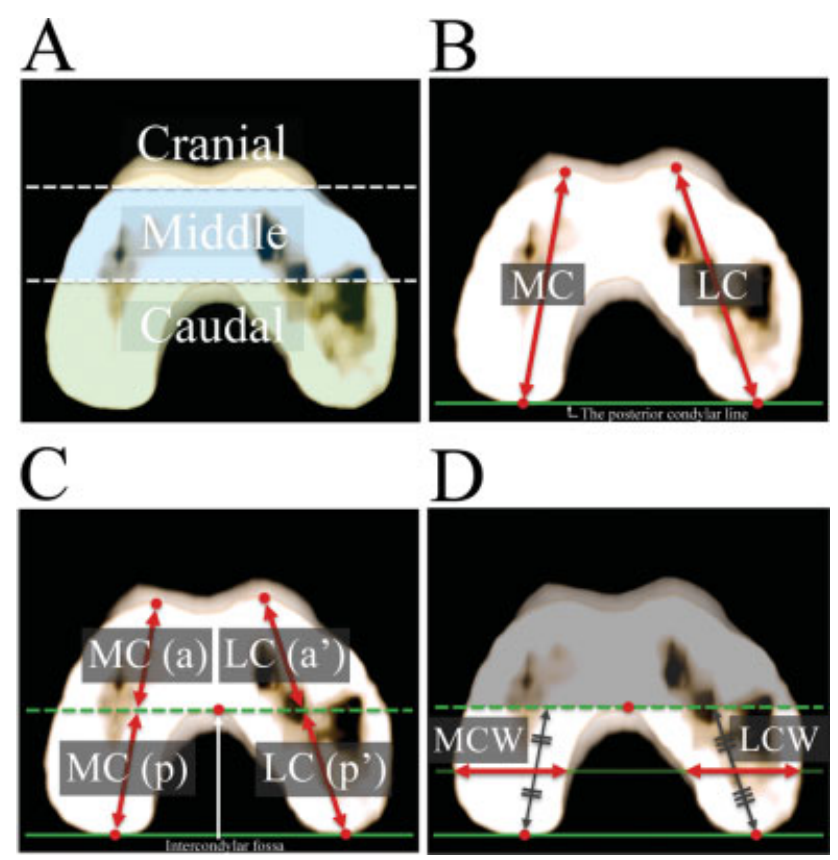

Fig. 3 Measurement values to evaluate the relationship between cranio-middle and caudal compartments. (A) Classification on transverse planes of the distal femur. Each transverse plane of the distal femur was divided into three compartments as follows: The 'cranial compartment', the area cranial to the bottom of the trochlear groove; the 'caudal compartment', the area caudal to the intercondylar fossa; the 'middle compartment', the area between the cranial and caudal compartments. (B) MC or LC: the distance between the apex of the medial or lateral trochlear ridge and the most caudal point of the medial or lateral femoral condyle respectively. (C) MC and LC were divided into two parts by the line that was drawn parallel to the caudal condylar line and passing through the intercondylar fossa (green dotted line) as follows: the anterior part (cranial compartment and middle compartment), MC (a) or LC (a'); and the posterior part (caudal compartment), MC (p) or LC (p'). (D) Medial or lateral condylar width (MCW or LCW): The width of the medial or lateral condyle at the level of the line that was drawn parallel to the posterior condylar line (green line) and passed through the midpoint of MC (p) or LC ( $\left.p^{\prime}\right)$.

11 stifles with grade $4 \mathrm{MPL})$. The mean age of the dogs was $1.0 \pm 1.7$ years (range: 3 months to 7 years), and the mean body weight of all evaluated dogs was $2.9 \pm 1.4 \mathrm{~kg}$ (range, $1.35-6.38 \mathrm{~kg}$ ). The dogs included 12 males (7 intact, 5 neutered) and 15 females ( 9 intact, 6 spayed). There were no significant differences in age, body weight and sex among groups. In the normal group, 16 limbs without any orthopaedic conditions of 13 dogs without any stifle pathology were included (both stifles were included in 3 dogs, each of which had a humeral fracture, cervical disease and carpal luxation). In the grade 2 MPL group, 10 limbs of 8 dogs were included; in the contralateral limb of the 8 dogs, 2 dogs had grade $2 \mathrm{MPL}, 2$ dogs had grade $4 \mathrm{MPL}$ and 4 dogs had conditions that met the exclusion criteria such as grade 1 or $3 \mathrm{MPL}$, lateral patellar luxation or hip and tarsal joint diseases. Both limbs were included in the 2 bilateral grade 2 MPL cases. In the grade 4 MPL group, 11 limbs of 8 dogs were included; in the contralateral limb, 2 dogs had grade 2 MPL, 3 dogs had grade 4 MPL and 3 dogs had conditions that met the exclusion criteria, as described in the section above. In 3 


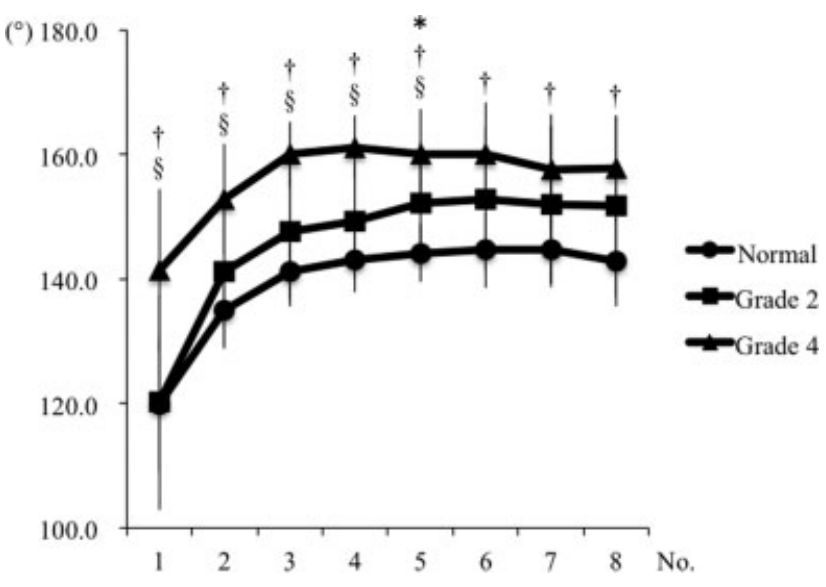

Fig. 4 Measurement values of sulcus angle. Mean values in the same transverse plane that have superscript reference symbols are significantly different among the groups $(p<0.05)$. (* normal vs. grade 2 $\mathrm{MPL} ; \dagger$, normal vs. grade $4 \mathrm{MPL}$; , grade $2 \mathrm{MPL}$ vs. grade $4 \mathrm{MPL}$ ). MPL, medial patellar luxation.

bilateral grade 4 MPL cases, both limbs were included. Two dogs with grade 2 and grade $4 \mathrm{MPL}$ on each stifle were included in this study.

\section{CT Measurements}

The SA was reliably determined on No. 1 to 8 out of 20 transverse planes in all groups (-Fig. 4). The SA in the more proximal part could not be reliably measured in some dogs because the trochlear groove was visually flat. In contrast, the TA could be determined in all 20 transverse planes (-Fig. 5). Other measurement values including the MTI, LTI, MC/LC, MC (a)/LC (a'), MC (a)/MC (p), LC (a)/LC (p), LC (a')/LC (p'), MC (p)/LC (p') and MCW/LCW were evaluated only on No.1 to 4 transverse planes in all groups ( - Tables 1 and 2), because caudal compartment could not be clearly identified on No.5 to 20 of transverse planes.

The SA was significantly higher in the grade 4 MPL group than in the normal group at all planes (-Fig. 4). In the distal half of the trochlear groove (from planes No.1 to 5), the SA was significantly higher (flat angle) in the grade 4 MPL group than in the grade 2 MPL group (-Fig. 4). At the mid-distal location of the groove (plane No.5), the SA was significantly higher (flat) in the grade $2 \mathrm{MPL}$ group than in the normal group (-Fig. 4). The MTI was also significantly lower in the grade $4 \mathrm{MPL}$ group than in the other groups at all examined planes (-Table 1). In contrast, there was no significant difference in the LTI among the groups ( $\mathbf{- T a b l e ~} \mathbf{1}$ ).

The TA decreased from the distal to the proximal trochlear groove in the grade $4 \mathrm{MPL}$ group, whereas it increased from the distal to the proximal trochlear groove in the other groups (-Fig. 5). On the No.1 to 6 transverse planes, the TA was significantly higher in the grade 4 MPL group than in the other groups. In contrast, on No.14 to 20 transverse planes, the TA was significantly lower in the grade $4 \mathrm{MPL}$ group than in the other groups, while on No.10 to 20 transverse planes, the TA was significantly higher in the grade 2 MPL group than in the normal group.

The MC/LC was generally lower in the grade 4 MPL group than in the other groups (-Table 2). The MC (a)/LC (a'), MC (a)/MC (p) and LC (a')/LC (p') were significantly lower in the grade 4 MPL group than in the other groups (-Table 2 ). In contrast, there were no significant differences in $\mathrm{MC}(\mathrm{p}) / \mathrm{LC}$ (p') among the groups (- Table 2). In addition, MCW/LCW generally showed no significant differences among the groups (-Table 2 ).

Taken together, no significant bone abnormalities were found in the distal femur in the grade 2 groups, with the exception of a poorly developed proximal medial trochlear

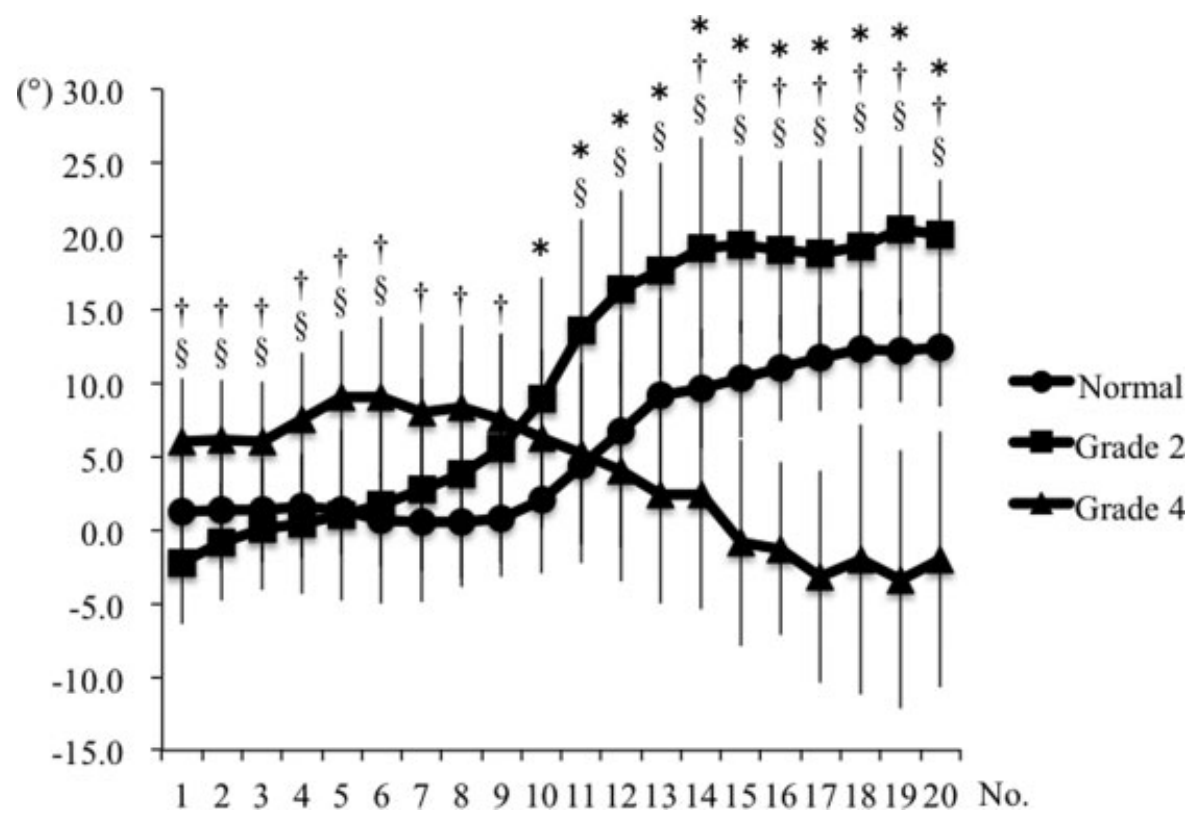

Fig. 5 Measurement values of trochlear angle. Mean values in the same transverse plane that have superscript reference symbols are significantly different among the groups $(p<0.05)$. ( ${ }^{*}$ normal vs. grade $2 \mathrm{MPL}$; $\dagger$, normal vs. grade $4 \mathrm{MPL}$; , grade $2 \mathrm{MPL}$ vs. grade $\left.4 \mathrm{MPL}\right)$. MPL, medial patellar luxation. 
Table 1 Measurement values for MTI and LTI

\begin{tabular}{|l|l|l|l|l|}
\hline & & Normal & Grade 2 & Grade 4 \\
\hline \multirow{4}{*}{ MTI } & No.1 & $29.1 \pm 5.8^{\S}$ & $30.8 \pm 9.7^{\S}$ & $12.9 \pm 9.2^{\dagger *}$ \\
\cline { 2 - 5 } & No.2 & $21.0 \pm 5.3^{\S}$ & $20.0 \pm 6.2^{\S}$ & $6.0 \pm 7.9^{\dagger *}$ \\
\cline { 2 - 5 } & No.3 & $17.2 \pm 3.8^{\S}$ & $15.5 \pm 8.5^{\S}$ & $3.7 \pm 4.9^{\dagger *}$ \\
\cline { 2 - 5 } & No.4 & $13.9 \pm 4.5^{\S}$ & $13.2 \pm 7.7^{\S}$ & $0.6 \pm 5.1^{\dagger *}$ \\
\hline \multirow{4}{*}{ LTI } & No.1 & $31.0 \pm 6.7$ & $29.0 \pm 8.8$ & $25.7 \pm 8.2$ \\
\cline { 2 - 5 } & No.2 & $24.0 \pm 4.5$ & $18.7 \pm 8.7$ & $21.3 \pm 8.0$ \\
\cline { 2 - 5 } & No.3 & $21.6 \pm 5.4$ & $16.9 \pm 8.0$ & $16.3 \pm 7.6$ \\
\cline { 2 - 5 } & No.4 & $23.1 \pm 4.8$ & $17.1 \pm 6.1$ & $18.1 \pm 7.4$ \\
\hline
\end{tabular}

Abbreviations: LTI, lateral trochlear inclination; MPL, medial patellar luxation; MTI, medial trochlear inclination.

*,t. § Mean values in the same row that have superscript reference symbols are significantly different between MPL grade groups $(p<0.05)$ $\left({ }^{*}\right.$, vs. normal; $\dagger$, vs. Grade 2 MPL; $\S$, vs. Grade 4 MPL).

Table 2 Measurement values for the distal femur

\begin{tabular}{|c|c|c|c|c|}
\hline & & Normal & Grade 2 & Grade 4 \\
\hline \multirow[t]{4}{*}{$\mathrm{MC} / \mathrm{LC}$} & No. 1 & $0.95 \pm 0.04$ & $1.00 \pm 0.04^{\S}$ & $0.91 \pm 0.07^{\dagger}$ \\
\hline & No.2 & $0.96 \pm 0.04^{\S}$ & $0.97 \pm 0.04^{\S}$ & $0.89 \pm 0.07^{* \dot{\dagger}}$ \\
\hline & No.3 & $0.96 \pm 0.03^{\S}$ & $0.97 \pm 0.04^{\S}$ & $0.90 \pm 0.07^{* \dagger}$ \\
\hline & No.4 & $0.95 \pm 0.03$ & $0.99 \pm 0.09^{\S}$ & $0.89 \pm 0.07^{\dagger}$ \\
\hline \multirow[t]{4}{*}{$M C(a) / L C\left(a^{\prime}\right)$} & No. 1 & $0.94 \pm 0.12^{\S}$ & $1.06 \pm 0.15^{\S}$ & $0.69 \pm 0.25^{* \dagger}$ \\
\hline & No.2 & $0.95 \pm 0.07^{\S}$ & $0.98 \pm 0.08^{\S}$ & $0.76 \pm 0.17^{* \dot{\dagger}}$ \\
\hline & No.3 & $0.95 \pm 0.05^{\S}$ & $0.97 \pm 0.07^{\S}$ & $0.83 \pm 0.12^{* \dagger}$ \\
\hline & No.4 & $0.94 \pm 0.04^{\S}$ & $1.00 \pm 0.13^{\S}$ & $0.83 \pm 0.10^{* \dagger}$ \\
\hline \multirow[t]{4}{*}{$M C(a) / M C(p)$} & No. 1 & $0.47 \pm 0.08^{\S}$ & $0.51 \pm 0.20^{\S}$ & $0.23 \pm 0.08^{* \dagger}$ \\
\hline & No.2 & $0.86 \pm 0.19^{\S}$ & $0.90 \pm 0.31^{\S}$ & $0.47 \pm 0.22^{* \dagger}$ \\
\hline & No.3 & $1.55 \pm 0.30^{\S}$ & $1.62 \pm 0.51^{\S}$ & $0.99 \pm 0.40^{* \dagger}$ \\
\hline & No.4 & $2.50 \pm 0.56^{\S}$ & $2.09 \pm 0.49^{\S}$ & $1.44 \pm 0.47^{* \dagger}$ \\
\hline \multirow[t]{4}{*}{$L C\left(a^{\prime}\right) / L C\left(p^{\prime}\right)$} & No. 1 & $0.49 \pm 0.11^{\S}$ & $0.49 \pm 0.19^{\S}$ & $0.34 \pm 0.06^{* 1}$ \\
\hline & No.2 & $0.89 \pm 0.19^{\S}$ & $0.92 \pm 0.30^{\S}$ & $0.58 \pm 0.15^{* 1}$ \\
\hline & No.3 & $1.58 \pm 0.28^{\S}$ & $1.71 \pm 0.50^{\S}$ & $1.14 \pm 0.31^{* \dagger}$ \\
\hline & No.4 & $2.60 \pm 0.60^{\S}$ & $2.10 \pm 0.36$ & $1.68 \pm 0.42^{*}$ \\
\hline \multirow[t]{4}{*}{$M C(p) / L C\left(p^{\prime}\right)$} & No. 1 & $0.96 \pm 0.02$ & $0.97 \pm 0.03$ & $0.98 \pm 0.03$ \\
\hline & No.2 & $0.97 \pm 0.02$ & $0.97 \pm 0.01$ & $0.97 \pm 0.04$ \\
\hline & No.3 & $0.97 \pm 0.02$ & $0.97 \pm 0.01$ & $0.98 \pm 0.02$ \\
\hline & No.4 & $0.97 \pm 0.01$ & $0.97 \pm 0.02$ & $0.98 \pm 0.03$ \\
\hline \multirow[t]{4}{*}{ MCW/LCW } & No.1 & $0.88 \pm 0.04$ & $0.95 \pm 0.08$ & $0.97 \pm 0.17$ \\
\hline & No.2 & $0.87 \pm 0.06^{\S}$ & $0.95 \pm 0.09$ & $1.01 \pm 0.20^{*}$ \\
\hline & No.3 & $0.90 \pm 0.05$ & $0.97 \pm 0.07$ & $0.99 \pm 0.15$ \\
\hline & No.4 & $0.88 \pm 0.09$ & $0.86 \pm 0.10$ & $0.89 \pm 0.13$ \\
\hline
\end{tabular}

Abbreviation: MPL, medial patellar luxation.

$*, \dagger$. § Mean values in the same row that have superscript reference symbols are significantly different between MPL grade groups $(p<0.05)$ ( ${ }^{*}$, vs. Normal; †, vs. Grade 2 MPL; §, vs. Grade 4 MPL). 
A
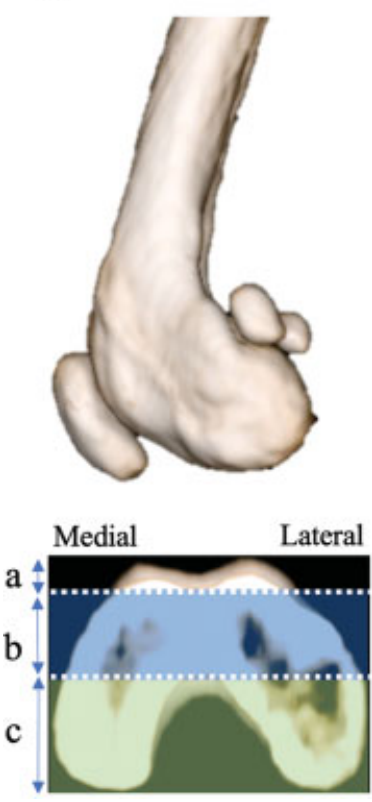

B
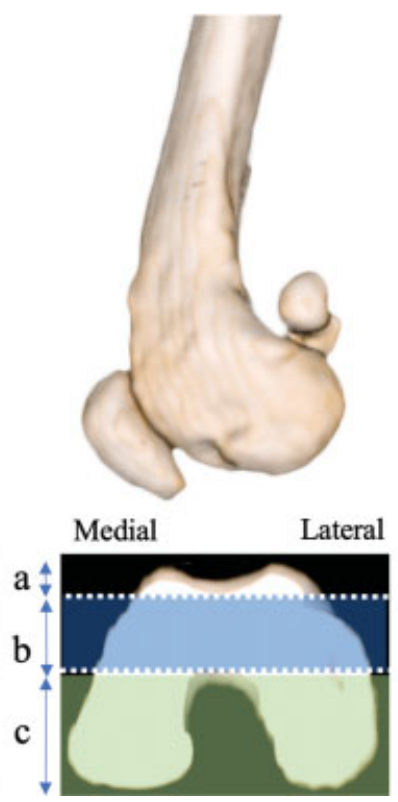

C
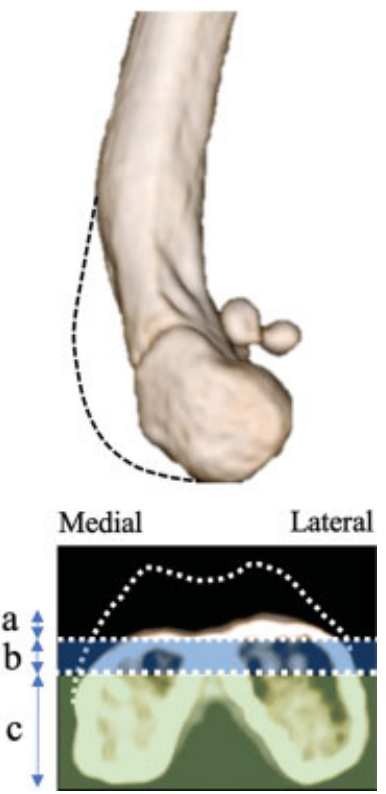

Fig. 6 Lateral views and No. 2 transverse planes of the distal femur in each group obtained by three-dimensional computed tomography. (A) Normal, (B) grade 2 MPL, (C) grade 4 MPL. a: Cranial compartment (trochlear groove), b: Middle compartment (bone under trochlear groove: sub-trochlear bone; blue zone), c: Caudal compartment (medial or lateral condyle; green zone). In the grade 4 MPL group, hypoplasia of the trochlear ridge (a: cranial compartment) and sub-trochlear bone (b: middle compartment; blue zone) was observed, and these abnormalities tended to be more severe on the medial side than on the lateral side (C). On the other hand, that hypoplasia of the caudal compartment (green zone) was not identified in any groups including the grade 4 MPL group (C). MPL, medial patellar luxation.

ridge (-Fig. 6). In contrast, significant hypoplasia of the trochlear groove was detected in the grade 4 MPL group, but significant hypoplasia of the medial condyle of the femur was not identified. Hypoplasia of the trochlear ridge (cranial compartment, - Fig. 6C) and the bone under the trochlear groove (sub-trochlear bone, middle compartment, blue zone in - Fig. 6C) was the primary anatomical abnormality observed in the grade $4 \mathrm{MPL}$ cases.

\section{Discussion}

In the present study, the morphology of the distal femur was evaluated objectively using 3D-CT imaging in dogs with and without MPL. Our results demonstrated that there were significant differences in several parameters in the grade $4 \mathrm{MPL}$ group compared with the normal and grade 2 MPL groups. It has been reported that hypoplasia of the trochlear groove occurs in dogs with severe MPL. ${ }^{1,3-5,15}$ However, there are no standardized methods to evaluate the depth of the trochlear groove because measurements can vary depending on the patient positioning and anatomical location. Recently, Petazzoni and colleagues proposed a method for trochlear groove depth measurement using CT in normal dogs. ${ }^{12,13}$ In the present study, sequential analysis of the distal femur was conducted including both the trochlear groove and the femoral condyle using 3D-CT imaging in dogs with grade 2 and 4 MPL. As a result, the morphology of the distal femur can be assessed at various locations, regardless of the positioning. The present study details the characteristics of the morphological abnormalities of the distal femur, and the results of the SA measurement indicate that hypoplasia of the trochlear groove was present only in the grade $4 \mathrm{MPL}$ group. The transverse CT images in the present study were images that were perpendicular to the line connecting point $a$ and $b$, not images that were perpendicular to the surface of trochlear groove. Therefore, SA that measured on the distal plane was tended to be affected by femoral morphology with strongly curved shape. However, the trends in SA among the groups could be identified because SA was measured under the same conditions. In addition, the results of MTI and LTI measurement indicated that the medial ridge of the trochlea was hypoplastic in the grade 4 MPL group. These findings indicate that the apparently shallow groove is actually due to hypoplasia of the ridges in grade $4 \mathrm{MPL}$.

In the current study, the TA results were unique in the grade 4 MPL group compared with the other groups (-Fig. 5). In human medicine, the TA has been used to examine the relationship between patellofemoral alignment and indices of knee pain and dysfunction. ${ }^{17}$ Furthermore, the TA has also been used to evaluate the degree of inclination of the trochlear groove to the medial or lateral direction. ${ }^{16,17}$ In the present study, the TA in the grade 4 MPL group indicated that the trochlear inclination to the medial direction was present toward the distal end of the femur. In contrast, the results of the MTI and the TA in the grade 2 MPL group reflected a poorly developed proximal medial trochlear ridge rather than trochlear inclination. In addition, no hypoplasia of the lateral ridge was observed in the grade 2 MPL group: this result suggested that repetitive luxation of the patella early in life may prevent normal development of the medial ridge in the proximal part. Future studies evaluating the effect of age and other grades of MPL are needed to confirm these findings. 
Most previous studies have demonstrated that the hypoplasia of the medial condyle of the femur occurs in severe MPL cases. ${ }^{1,3-5}$ However, to the best of our knowledge, no published study has objectively evaluated the morphology of the femoral condyle with severe MPL. In the present study, the morphology of the distal femur was evaluated in three compartments. The results from MC (p)/LC ( $\left.p^{\prime}\right)$ and MCW/LCW showed no hypoplasia of the caudal compartment in any groups, including the grade $4 \mathrm{MPL}$ group. In contrast, the results of MC (a)/MC (p), LC (a')/LC (p') showed that hypoplasia of the cranial and middle compartments was the primary anatomical abnormality in the grade $4 \mathrm{MPL}$ group ( - Fig. 6). In addition, the results of MTI, MC/LC and MC (a)/LC ( $\left.a^{\prime}\right)$ indicated that the abnormalities tended to be more severe on the medial side than on the lateral side.

In previous studies, articulation of the patella within the trochlear groove during development has been considered to influence the depth of the trochlear groove. ${ }^{1}$ However, the results of this study indicated that the bone abnormality in the distal femur associated with MPL was hypoplasia of the trochlear ridge and sub-trochlear bone, rather than the shallow trochlear groove (-Fig. 6). Therefore, patellar tracking during development may be essential for the normal development of both the cranial and middle compartments of the distal femur. Surgical procedures to treat MPL aim to correct malalignment of the quadriceps mechanism and generally involve a combination of soft tissue reconstruction, trochleoplasty and lateral transposition of the tibial tuberosity. ${ }^{1-4}$ Trochleoplasty is used to prevent reluxation of the patella and is intended to improve the depth of the shallow trochlear groove and increase the contact area between the patella and the trochlear groove. However, indications for this procedure mainly depend on the surgeon's experiences. ${ }^{19,20}$ These results could be useful to determine the indication for trochleoplasty, and it may be necessary to take the trochlear inclination to the medial direction into consideration in the distal half of the trochlear groove when trochleoplasty is performed in the grade $4 \mathrm{MPL}$ group. Furthermore, trochleoplasty should be performed carefully to avoid the iatrogenic fracture of the distal femur for dogs with grade 4 MPL because these dogs often have hypoplasia of the cranial and middle compartments of the distal femur (-Fig. 6).

The current study evaluated Toy Poodles with only grades 2 and 4 MPL. Most previous radiographic studies that have examined bone morphologies of the distal femur associated with MPL have included various breeds and grades. ${ }^{21-23}$ To decrease the variability associated with anatomic variations among breeds, we focused on a single breed that is known to have a breed predilection for MPL. ${ }^{3,24,25}$ Future studies will be necessary to understand the differences in pathology in various breeds by examining more dogs of other breeds. Furthermore, we did not evaluate the bone morphology in the dogs with grades 1 and $3 \mathrm{MPL}$ due to the small sample size during the investigation period. Thus, if the parameters were evaluated in dogs with grades 1 and $3 \mathrm{MPL}$, the understanding of bone deformities of the distal femur associated with MPL will be improved.
The limitation of this study was the inclusion of young immature dogs to evaluate bone deformities of the distal femur. Grade $4 \mathrm{MPL}$ cases are often treated at very young age. When a study on bone deformity is conducted using grade 4 MPL cases, data on very young dogs are valuable, clinically relevant, therefore should be included. Thus, younger dogs were included in normal and grade 2 MPL groups as well as in grade 4 MPL group and the groups were set up so that there was no significant difference in the age of month among the groups. The present study also included some dogs with bilateral MPL. However, it was confirmed that the presence of contralateral MPL does not have a significant effect on bone morphology in our preliminary and previous published studies. ${ }^{14}$ In the present study, CT was used to evaluate the morphology of the trochlear groove. CT can only assess osseous trochlear morphology. In contrast, MRI studies can reveal chondral morphology. Therefore, it might be preferable to use MRI to accurately determine the shape of the trochlear groove.

In conclusion, hypoplasia of the cranial and middle compartments of the distal femur was the primary morphological abnormality in Toy Poodles with grade 4 MPL. In addition, no significant hypoplasia of the caudal compartment was identified in any of the dogs evaluated in this study. In contrast, no significant bone deformities, with the exception of a poorly developed proximal medial trochlear ridge, were observed in the grade 2 MPL group. The results of the present study provide new findings related to bone deformities in MPL and contribute to a better understanding of the pathophysiology and treatment strategy for MPL in dogs.

\section{Author's Contributions}

All authors conceptualized the study. They drafted, revised, approved the manuscript and are publically accountable for relevant content. S.Y., K.E., M.S., K.A., T.N. and K.H. designed the study; S.Y. and K.T. acquired the data; S.Y., K.E., K.T. and K.H. analyzed and interpreted the data.

Conflict of interest

None declared.

\section{References}

1 Schulz KS, Hayashi K, Fossum TW. Diseases of the joints. In: Fossum TW, ed. Small Animal Surgery. 5th edition St Louis: Mosby Elsevier; 2019:1134-1279

2 Vasseur PB. Stifle joint. In: Slatter D, ed. Textbook of Small Animal Surgery. 3rd edition Philadelphia: Saunders; 2003:2090-2132

3 DeCamp CE, Johnson SA, Dejardin LM, Schaefer SL. The stifle joint. In: DeCamp CE, Johnson SA, Dejardin LM, Schaefer SL, eds. Handbook of Small Animal Orthopedics and Fracture Repair. 5th edition Philadelphia: Elsevier; 2016:597-669

4 Roush JK. Canine patellar luxation. Vet Clin North Am Small Anim Pract 1993;23(04):855-868

5 Hulse DA. Pathophysiology and management of medial patellar luxation in the dog. Vet Med Small Anim Clin 1981;76(01):43-51

6 Tomlinson J, Fox D, Cook JL, Keller GG. Measurement of femoral angles in four dog breeds. Vet Surg 2007;36(06):593-598

7 Farrell M. The stifle. In: Arthurs G, Brown G, Pettitt R, eds. BSAVA Manual of Canine and Feline Musculoskeletal Disorders. 2nd edition Quedgeley: BSAVA; 2018:404-446 
8 Meier HT, Biller DS, Lora-Michiels M, Hoskinson JJ. Additional radiographic views of the pelvis and pelvic limb in dogs. Compend Contin Educ Pract Vet 2001;23:871-874

9 Meyer H, Kaiser S, Waibl H, Brunnberg L. Radiological parameters for preoperative planning of the surgical correction of canine congenital patellar luxation (part 1). Kleintierpraxis 2002; 47:65-70

10 Meyer H, Kaiser S, Waibl H, Brunnberg L. Radiological parameters for preoperative planning of the surgical correction of canine congenital patellar luxation (part 2). Kleintierpraxis 2002; 47:129-138

11 Towle HA, Griffon DJ, Thomas MW, Siegel AM, Dunning D, Johnson A. Pre- and postoperative radiographic and computed tomographic evaluation of dogs with medial patellar luxation. Vet Surg 2005;34(03):265-272

12 Petazzoni M, De Giacinto E, Troiano D, Denti F, Buiatti M. Computed tomographic trochlear depth measurement in normal dogs. Vet Comp Orthop Traumatol 2018;31(06):431-437

13 Nicetto T, Longo F, Contiero B, Isola M, Petazzoni M. Computed tomographic localization of the deepest portion of the femoral trochlear groove in healthy dogs. Vet Surg 2020;49(06): 1246-1254

14 Yasukawa S, Edamura K, Tanegashima K, et al. Evaluation of bone deformities of the femur, tibia, and patella in Toy Poodles with medial patellar luxation using computed tomography. Vet Comp Orthop Traumatol 2016;29(01):29-38

15 Singleton WB. The surgical correction of stifle deformities in the dog. J Small Anim Pract 1969;10(02):59-69

16 Stefanik JJ, Zumwalt AC, Segal NA, Lynch JA, Powers CM. Association between measures of patella height, morphologic features of the trochlea, and patellofemoral joint alignment: the MOST study. Clin Orthop Relat Res 2013;471(08): 2641-2648
17 Kalichman L, Zhu Y, Zhang Y, et al. The association between patella alignment and knee pain and function: an MRI study in persons with symptomatic knee osteoarthritis. Osteoarthritis Cartilage 2007;15(11):1235-1240

18 Carrillon Y, Abidi H, Dejour D, Fantino O, Moyen B, Tran-Minh VA. Patellar instability: assessment on MR images by measuring the lateral trochlear inclination-initial experience. Radiology 2000; 216(02):582-585

19 Linney WR, Hammer DL, Shott S. Surgical treatment of medial patellar luxation without femoral trochlear groove deepening procedures in dogs: 91 cases (1998-2009). J Am Vet Med Assoc 2011;238(09):1168-1172

20 Hansen JSO, Lindeblad K, Buelund L, Miles J. Predicting the need for trochleoplasty in canine patellar luxation using pre- and intraoperative assessments of trochlear depth. Vet Comp Orthop Traumatol 2017;30(02):131-136

21 Mortari AC, Rahal SC, Vulcano LC, da Silva VC, Volpi RS. Use of radiographic measurements in the evaluation of dogs with medial patellar luxation. Can Vet J 2009;50(10):1064-1068

22 Bound N, Zakai D, Butterworth SJ, Pead M. The prevalence of canine patellar luxation in three centres. Clinical features and radiographic evidence of limb deviation. Vet Comp Orthop Traumatol 2009;22(01):32-37

23 Barnes DM, Anderson AA, Frost C, Barnes J. Repeatability and reproducibility of measurements of femoral and tibial alignment using computed tomography multiplanar reconstructions. Vet Surg 2015;44(01):85-93

24 Hodgman SFJ. Abnormalities and defects in pedigree dogs-I. An investigation into the existence of abnormalities in pedigree dogs in the British Isles. J Small Anim Pract 1963;4:447-456

25 LaFond E, Breur GJ, Austin CC. Breed susceptibility for developmental orthopedic diseases in dogs. J Am Anim Hosp Assoc 2002; 38(05):467-477 